\title{
Bioinformatics Analysis of Merkel Cell Carcinoma to Identify Critical Genes and their Validation
}

\author{
Himanshu Kumar \\ Department of Biotechnology \\ Delhi Technological University \\ Delhi, INDIA \\ himanshu97kumar@gmail.com
}

\author{
*Yasha Hasija \\ Department of Biotechnology \\ Delhi Technological University \\ Delhi, INDIA \\ yashahasija@gmail.com \\ *Corresponding Author
}

\begin{abstract}
Merkel Cell Carcinoma (MCC) is a destructive form of neuroendocrine carcinoma that represents the 2nd highest cause of skin cancer related deaths despite being a relatively rare form of cancer. Different risk factors have been associated with MCC such as advancement age, immunosuppression, and ultraviolet light exposure, polyomavirus infection and a current, concurrent or previous diagnosis of Chronic Lymphocytic Leukaemia (CLL). However, the exact mechanism that leads to MCC and its inherent biology is yet to be fully researched and explored. The current treatments for $\mathrm{MCC}$ are a combination of surgery and radiation or chemo-radiation. So far, only two drugs have been approved by FDA for the treatment of MCC, with both drugs being immune checkpoint inhibitors. However, the drug approval from FDA was accelerated based on promising results from a relatively small number of patients, which means that the more research and trials are undergoing currently considering the fact that a very small cohort was used for clinical trials. Presently there are no biomarkers indicative of MCC and additional research is essential. With the advent of bioinformatics and microarray technology, in silico approaches pertaining to 'omics' data analysis, shall allow identification of hub genes and miRNA to broaden our understanding of MCC biology and their clinical utility.
\end{abstract}

Keywords- Merkel Cell Carcinoma, Microarray, Bioinformatics Analysis, Gene Analysis

\section{INTRODUCTION}

Merkel Cell Carcinoma (MCC) is destructive form of neuroendocrine carcinoma whose manifestation of occurrence have been on a rise in recent years. It represents the 2nd highest cause of skin cancer related deaths despite being a relatively rare form of cancer [1]. A series of ultrastructural studies revealed the similarity of these cancer cells to that of Merkel Cells (a type of mechanoreceptor cells present in the skin), which led to the renaming of these cells from trabecular carcinoma to Merkel Cell Carcinoma [2]. Different risk factors have been associated with MCC such as advancement in age, immunosuppression, and exposure to UV. A link between MCC and PyV infection has also been established. An existing, concurrent or even a previous diagnosis of Chronic Lymphocytic Leukaemia (CLL) is more frequent in people with MCC [3]. However, the exact biology of MCC and its carcinogenesis is yet to be fully explored.

The current treatments for MCC are surgery along with radiation or chemo-radiation. The first FDA approved drug for MCC was avelumab based on a clinical trial of eightyeight patients diagnosed with metastatic MCC who had formerly undergone chemo-radiation for the same [4]. A second drug was approved in the beginning of 2019, called pembrolizumad. Both these drugs are immune checkpoint inhibitors [5]. As a result, there has been a complete sea change in the management of MCC patients in the last two years. Prior to using immunotherapy as a first line of treatment, MCC patients were treated just like any other individual with a neuroendocrine tumor. However, the current approval from FDA has been an accelerated one, based on promising results from a relatively small number of patients, which means that the more research and trials are undergoing currently considering the fact that a very small cohort was used for clinical trials. Although, 56\% of the drug recipients have shown a positive response, many stopped using the drug due to severe side effects. A further issue is that many patients with MCC may not be ideal candidates for immunotherapy because their immune systems have been suppressed - due to organ/graft transplantation, lymphoma or HIV [5].

At the moment, there is a lack of definitive biomarkers that could aid in a more effective selection of patients for new therapies, thereby creating a crucial need for further research. The present study is therefore an attempt to identify key genes and microRNAs in MCC by various in silico methodologies.

\section{MATERIALS AND METHODS}

\section{A. Microarray data processing and screening for DEGs} and DEmiRs

In this study, the expression profile GSE39612 from the GEO database was used to identify DEGs. The series GSE39612 provides transcription data of 30 tumors from 27 different patients from which samples of MCC and normal skin were acquired. DEGs were identified by using Subio platform (Subio Inc., Japan) where a p-value of $<0.05$ and $|\mathrm{FC}|>1.5$ was considered as the cut-off. Furthermore, the series GSE45146 was used to identify DEmiRNAs between normal skin samples and MCC. This analysis was performed by using GEO2R tool where DEmiRNAs are considered on the basis of statistical measures that met adjusted P-value $(\operatorname{adj} . \mathrm{P})<0.01$ and $\mid \log$ fold change $\mid>1.5$ of miRNAs.

\section{B. Pathway and GO enrichment analysis of DEGs}

To understand the functional changes of the DEGs, their cellular components (CC), biological processes (BP) and molecular function (MF) were analysed by DAVID [6]. Only GO terms with $\mathrm{p}$-value $<0.05$ and count $>2$ were measured statistically important. 
Enrichment of pathways was also performed by using DAVID. The DEGs were used to perform a Fisher's exact test followed by multiple test correction using BH's False Discovery Rate correction. A revised p-value of $<0.05$ was considered statistically important to determine the overrepresentation of certain biological pathways.

\section{PPI network construction}

Cytoscape is used along with STRING database [7] to construct a PPI network. Only query proteins were displayed and a lowest required interaction score of 0.4 (medium confidence) was kept as the primary parameter. Subsequently, significant cluster screening was done by using another Cytoscape plugin, MCODE, using their pre-set cut- off criteria [8].

\section{Identification of hub genes using cytoHubba}

Five algorithms, namely, MCC, MNC, EPC, Degree and EcCentricity were determined using cytoHubba plug-in to categorize hub genes from the PPI network. The overlapped genes of the five algorithms mentioned above were further enriched in terms of KEGG pathways, GO molecular functions, GO biological processes and GO cellular components using DAVID 6.8

\section{E. Target gene prediction of DE microRNA}

To identify putative target genes of the DEmiRs, three online resources were used. TargetScan Human version 7.2 and miRDB were used to identify predictive miRNA-gene pairs while miRTarBase was used to identify experimentally validated miRNA-gene pairs.

\section{F. Construction and analysis of DEG-DEmiR network}

The DEGs and DEmiRs were used towards constructing a dysregulation network using Cytoscape. Using Network Analyser tool of Cytoscape, the above network was analysed for the purpose of finding hub miRNAs. A degree cut-off of $>20$ was set as the criteria. Hub node annotation in terms of GO molecular function, GO biological process, GO cellular components and KEGG pathway were again performed by DAVID.

\section{RESULTS}

\section{A. Identification of DEGs and DEmiRs from datasets}

To find DEGs between normal skin samples and MCC, GSE39612 series was used in Subio platform. DEGs are evaluated by fold change of 1.5 and $p$-value $<0.05$. A total of 449 genes were recognized which included 285 downregulated genes and 164 upregulated genes. Whereas, the series GSE45146 was used to identify DEmiRNAs between normal skin samples and MCC using GEO2R. DEmiRNAs are considered on the basis of statistical measures that met adjusted P-value (adj. P) $<0.01$ and $\mid \log$ fold change| $>1.5$ of miRNAs. A total of 17 DEmiRNAs were identified which included 1 upregulated miRNA and 16 downregulated miRNAs.

\section{B. Functional enrichment analysis of DEGs}

To recognize the function of the DEGs, their biological processes (BP), cellular components (CC) and molecular function (MF) were analysed by DAVID. The DEGs were mainly enhanced in oxidation-reduction process (GO: 0055114), keratinocyte differentiation (GO: 0030216), epidermis development (GO: 0008544) and positive regulation of cell proliferation (GO: 0008284) in the BP group. In CC group, the DEGs were mostly enriched in plasma membrane (GO: 0005886), cytoplasm (GO: 0005737) and extracellular space (GO: 0005615). Finally, in the MF group, the genes enriched in calcium ion binding (GO: 0005509), protein homo-dimerization activity (GO: 0042803) and structural molecule activity (Table 3.1, 3.2). Meanwhile, significantly enriched pathways highlighted were cAMP signalling pathway (hsa04024), Calcium signalling pathway (hsa04020), Oocyte meiosis (hsa04114), Arachidonic acid metabolism (hsa00590), Vascular smooth muscle contraction (hsa04270), Inflammatory mediator regulation of TRP channels (hsa04750), GnRH signalling pathway (hsa04912), p53 signalling pathway (hsa04115), Aldosterone synthesis and secretion (hsa04925) and HIF-1 signalling pathway (hsa04066).

TABLE I. A TABULAR REPRESENTATION OF ANALYSIS OF SIGNIFICANT ENRICHMENT GO TERMS OF DEGS PRESENT IN MCC

\begin{tabular}{|l|l|l|l|l|}
\hline \multicolumn{1}{|c|}{ Term } & \multicolumn{1}{|c|}{ Description } & \multicolumn{1}{c|}{ Count } & \multicolumn{1}{c|}{ P Value } & \multicolumn{1}{c|}{ Genes } \\
\hline Biological Process & & & & \\
\hline GO:0045944 & $\begin{array}{l}\text { RNA polymerase II promoter positive regulation of } \\
\text { transcription }\end{array}$ & 46 & $494 \mathrm{E}-05$ & $\begin{array}{l}\text { FGFR,HLF,IL18,FOXM1, } \\
\text { F2RL1,SOX2,BEX1,PAX6, }\end{array}$ \\
\hline GO:0008284 & cell proliferation positive regulation & 29 & $1.53 \mathrm{E}-05$ & FGFR2,KRT6A,CCK,IL6ST, \\
\hline GO:0006366 & transcription from RNA polymerase II promoter & 27 & 0.000471 & $\begin{array}{l}\text { HLF,FOXM1,SOX2,PAX6, } \\
\text { PAX5,TP63,EHF,MYBL1, }\end{array}$ \\
\hline GO:0055114 & oxidation-reduction process & 25 & 0.012791 & $\begin{array}{l}\text { ME1,TYRP1,HSD3B1, } \\
\text { CYP1B1,EGLN3,BBOX1, }\end{array}$ \\
\hline GO:0008544 & epidermis development & 24 & $5.00 E-18$ & $\begin{array}{l}\text { KLK7,KLK5,LCE2B,KRT31, } \\
\text { GJB5,CDSN,SCEL }\end{array}$ \\
\hline GO:0007155 & cell adhesion & 24 & 0.001142 & $\begin{array}{l}\text { CLCA2,TNXB,CYP1B1, } \\
\text { SLURP1,ADAM23,CTNND2 }\end{array}$ \\
\hline GO:0045893 & DNA-templated, Positive regulation of transcription & 24 & 0.004885 & $\begin{array}{l}\text { KLF5,SOC1,FOXM1,PSRC1, } \\
\text { SOX2,PAX6,IGF1, }\end{array}$ \\
\hline $\begin{array}{c}\text { Cellular } \\
\text { Components }\end{array}$ & Cytoplasm & & & \\
\hline GO:0005737 & plasma membrane & 158 & 0.000572 & $\begin{array}{l}\text { PALMD,DCN,KRT5,NCAPG, } \\
\text { HJURP,POU2F3, }\end{array}$ \\
\hline GO:0005886 & 135 & $5.74 E-05$ & $\begin{array}{l}\text { TBC1D30,FCER1A,KLF5, } \\
\text { VAV3,ADAM23,IL1RN, }\end{array}$ \\
\hline
\end{tabular}




\begin{tabular}{|l|l|l|l|l|}
\hline GO:0070062 & extracellular exosome & 126 & $1.19 \mathrm{E}-12$ & $\begin{array}{l}\text { CLEC3B,FBLN5,SPRR1B, } \\
\text { AOX1,DSC2,DSC1,CP }\end{array}$ \\
\hline GO:0005829 & Cytosol & 99 & 0.014265 & $\begin{array}{l}\text { CENPE,CAPN3,RGS13,SCG, } \\
\text { AOX1,RAB38,HPGD }\end{array}$ \\
\hline GO:0005576 & extracellular region & 90 & $5.16 \mathrm{E}-14$ & $\begin{array}{l}\text { SCGN,OMD,FBLN1,CD55, } \\
\text { CCL14,NPY,SFRP1, }\end{array}$ \\
\hline GO:0005615 & extracellular space & 85 & $3.42 \mathrm{E}-16$ & $\begin{array}{l}\text { FBLN1,OMD,CCL14,NPY, } \\
\text { SFRP1,CLEC3B,FBLN5, }\end{array}$ \\
\hline GO:0005887 & $\begin{array}{l}\text { integral component of plasma } \\
\text { membrane }\end{array}$ & 47 & 0.022283 & $\begin{array}{l}\text { P2RY14,NTRK2,KCNH6, } \\
\text { CLDN1,PDGFRA,TM4SF1, }\end{array}$ \\
\hline GO:0005794 & Golgi apparatus & 28 & 0.098523 & $\begin{array}{l}\text { NPY,DUSP26,ATP8A2, } \\
\text { RAB38,PERP,KIF20A }\end{array}$ \\
\hline $\begin{array}{l}\text { Molecular } \\
\text { Function }\end{array}$ & & & & \\
\hline GO:0005515 & protein binding & 249 & 0.001438 & $\begin{array}{l}\text { KIF18A,CENPF,DPYSL4, } \\
\text { CENPE,DACH1,ISL1, }\end{array}$ \\
\hline GO:0005509 & calcium ion binding & 48 & $1.12 \mathrm{E}-09$ & $\begin{array}{l}\text { DSC2,LRP8,DSC1,NCAN, } \\
\text { DST,PROS1,CACNA1A, }\end{array}$ \\
\hline GO:0042803 & protein homo-dimerization activity & 32 & 0.002538 & $\begin{array}{l}\text { FBLN5,KCNN2,NTRK2, } \\
\text { PDGFRA,CLIP1,CRRBA2, }\end{array}$ \\
\hline GO:0005198 & structural molecule activity & 27 & $5.44 \mathrm{E}-10$ & $\begin{array}{l}\text { KRT15,KRT14,CLDN1,DSP, } \\
\text { CSTA,ADD2, }\end{array}$ \\
\hline GO:0001077 & $\begin{array}{l}\text { transcriptional activator activity, } \\
\text { RNA polymerase II core promoter }\end{array}$ & 21 & $1.84 \mathrm{E}-06$ & $\begin{array}{l}\text { POU4F2,POU3F2,ZNF750, } \\
\text { POU4F1,KLF4,PITX2, }\end{array}$ \\
\hline GO:0005200 & structural constituent of cytoskeleton & 19 & $2.10 \mathrm{E}-10$ & $\begin{array}{l}\text { KRT14,DSP,ACTL6B,NEFH, } \\
\text { KRT2,NEFL,NEFM, }\end{array}$ \\
\hline GO:0008201 & heparin binding & 14 & 0.000178 & FGFR2,TNXB,ECM2,GREM2,APLP1,PRELP \\
\hline
\end{tabular}

TABLE II. A TABULAR REPRESENTATION OF SIGNIFICANT KEGG PATHWAYS IDENTIFIED FOR THE DEGS IN THIS DATASET

\begin{tabular}{|c|c|c|c|}
\hline Term & Description & $\mathrm{p}$-Value & Genes \\
\hline hsa04024 & cAMP signaling pathway & 0.006951985 & $\begin{array}{l}\text { ATP2B2,ADCY1,PLD1,VAV3, } \\
\text { NPY,CALML3,PLN, }\end{array}$ \\
\hline hsa04020 & Calcium signaling pathway & 0.008673811 & $\begin{array}{l}\text { EGFR,GNAL,ATP2B2,AGTR1, } \\
\text { ADCY1,CALML3,PLN, }\end{array}$ \\
\hline hsa05146 & Amoebiasis & 0.007513759 & $\begin{array}{l}\text { GNAL,IL1R2,ARG1,LAMB4, } \\
\text { ADCY1,SERPINB2, }\end{array}$ \\
\hline hsa04114 & Oocyte meiosis & 0.009824526 & $\begin{array}{l}\text { CCNE2,ADCY1,CALM3,BUB1, } \\
\text { FBXO5,IGF1,AURKA, }\end{array}$ \\
\hline hsa00590 & Arachidonic acid metabolism & 0.001161382 & $\begin{array}{l}\text { AKR1C3,PLA2G4A,PTGIS, } \\
\text { ALOX15B,PLA2G2A,EPHX2, }\end{array}$ \\
\hline hsa05214 & Glioma & 0.001693434 & $\begin{array}{l}\text { EFGR,CDKN2A,CALML3, } \\
\text { PDGFRA,IGF1,CAMK2B }\end{array}$ \\
\hline hsa04750 & $\begin{array}{llll}\begin{array}{l}\text { Inflammatory mediator regulation of } \\
\text { channels }\end{array} & \text { TRP } \\
\end{array}$ & 0.016071416 & $\begin{array}{l}\text { ADCY1,PLA2G4A,CALML3, } \\
\text { F2RL1,IGF1,CAMK2B, }\end{array}$ \\
\hline hsa04270 & Vascular smooth muscle contraction & 0.037827198 & $\begin{array}{l}\text { ACTG2,AGTR1,ADCY1, } \\
\text { PLA2G4A,CALML3,CALD1, }\end{array}$ \\
\hline hsa04115 & p53 singaling pathway & 0.008943032 & $\begin{array}{l}\text { CCNE2,CDKN2A,RRM2, } \\
\text { RPRM,IGF1,PERP,GTSE1 }\end{array}$ \\
\hline hsa04925 & Adosterone synthesis and secretion & 0.021401112 & $\begin{array}{l}\text { AGTR1,ADCY1,HSDB1, } \\
\text { CALML3,CAMK2B,CALML5, }\end{array}$ \\
\hline hsa04912 & GnRH signaling pathway & 0.035359873 & $\begin{array}{l}\text { EFGR,ADCY1,PLA2G4A, } \\
\text { PLD1,CALML3,CAMK2B, }\end{array}$ \\
\hline hsa04066 & HIF-1 signaling pathway & 0.044130154 & $\begin{array}{l}\text { EFGR,TF,EGLN3,ENO2,IGF1, } \\
\text { CAMK2B,PIK3R1 }\end{array}$ \\
\hline hsa04913 & Ovarian steroidogenesis & 0.009751094 & $\begin{array}{l}\text { AKR1C3,ADCY1,PLA2G4A, } \\
\text { HSD3B1,CYP1B1,IGF1 }\end{array}$ \\
\hline hsa04924 & Renin secretion & 0.028291683 & $\begin{array}{l}\text { AGTR1,CLCA2,CLCA4, } \\
\text { CALML3,CALML5,AQP1 }\end{array}$ \\
\hline hsa03320 & PPAR signaling pathway & 0.033623759 & $\begin{array}{l}\text { CPTIB,NPY,LEPR,ADIPOQ, } \\
\text { ACSL6,ACSBG1 }\end{array}$ \\
\hline hsa04920 & Adipocytokine signaling pathway & 0.039542173 & $\begin{array}{l}\text { CPT1B,NPY,LEPR,ADIPOQ, } \\
\text { ACSL6,ACSBG1 }\end{array}$ \\
\hline hsa05218 & Melanoma & 0.041647285 & $\begin{array}{l}\text { EFGR,CDKN2A,PDGFRA,IGF1, } \\
\text { CDH1,PIK3R1 }\end{array}$ \\
\hline hsa00350 & Tyrosine metabolism & 0.013828406 & $\begin{array}{l}\text { DCT,TYRP1,AOX1,ADH1B, } \\
\text { ALDH3B2 }\end{array}$ \\
\hline hsa00565 & Ether lipid metabolism & 0.032014452 & $\begin{array}{l}\text { PLA2G4A,PLD1,PLA2G2A, } \\
\text { PAFAH1B1,PLPP3 }\end{array}$ \\
\hline hsa01212 & Fatty acid metabolism & 0.039315876 & $\begin{array}{l}\text { CPT1B,FLOVL2,SCD5,ACSL6, } \\
\text { ACSBG1 }\end{array}$ \\
\hline hsa04614 & Renin-angiotensin system & 0.022816032 & AGTR1,CPA3,CMA1,CTSG \\
\hline
\end{tabular}




\section{Construction of PPI network and its module analysis}

With the help of STRING database on Cytoscape, PPI network was constructed with a pre-set criteria of displaying only query proteins and a minimum required interaction score of 0.4 as primary parameters. A network of 448 nodes and 2190 edges were included in the DEGs network. Subsequently, significant module selection was done by using, MCODE, using their pre-set cut- off criteria. The clusters were primarily enriched in cell cycle checkpoints, cell cycle, structural constituent of epidermis, regulation of cell cycle, cornified envelope, cell-cell adhesion and immune system.

\section{Identification of Hub Genes and Pathways through analysis of PPI network}

CytoHubba was employed to observe the intersection of five algorithms (degree, MCC, MNC, EPC and EcCentricity) to identify the hub genes present in the network. As seen from Figure 3.1, 16 genes were found in the intersection of the five algorithms. These genes include: AURKA, FLG, KIF4A, DSG1, PKP1, LOR, BUB1, CDC6, CENPE, CXCR4, FOXM1, CXCL12, CDSN, DSG3, IVL and DSP.

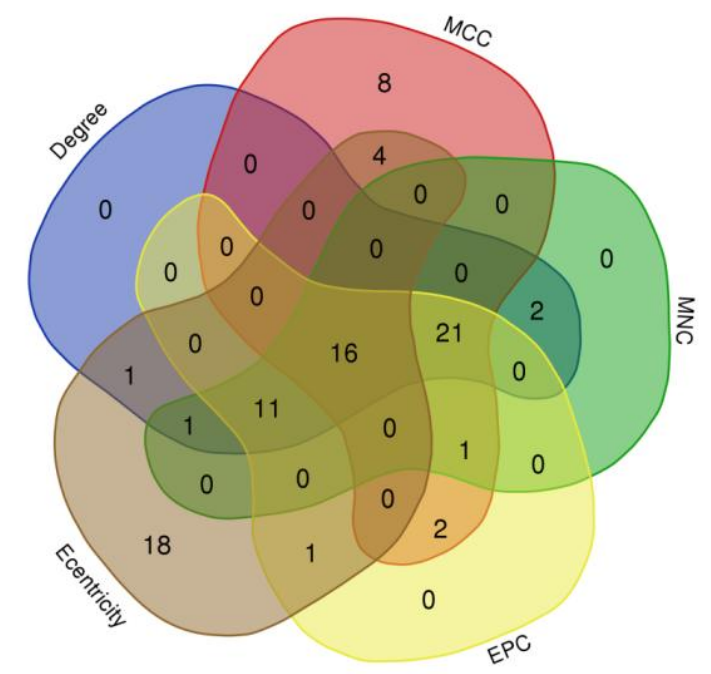

Fig. 1. Representation of Venn plot by intersecting 5 algorithms to identify significant hub genes. Different algorithms are represented by different colours

The 16 hubs genes included 7 upregulated and 9 downregulated genes and were used towards constructing a network with 34 edges and 16 nodes. A network of these 16 hub genes revealed that the upregulated and downregulated genes were part of two individual clusters, as shown in Figure 3.2. A STRING enrichment analysis (GO components, GO processes, GO functions, KEGG pathways and Reactome pathways) was performed for the hub genes, keeping the FDR $<0.05$. In the GO components group, the genes were mostly enriched in cornified envelope (GO.0001533), desmosome (GO.0030057), cytoskeletal part (GO.0044430), plasma membrane (GO.0005886) and cytosol (GO.0005829). In the GO process group, most genes were enriched in programmed cell death (GO.0012501), cell differentiation (GO.0030154), animal organ development (GO.0048513), cornification (GO.0070268), anatomical structure development (GO.0048856) and system development (GO.0048731). Further, genes were mainly enriched in protein binding (GO.0005515) and binding (GO.0005488) in the GO functions group. For pathway enrichment, results from both KEGG and Reactome were considered. Eight KEGG pathways were highlighted which included Cell cycle (hsa04110), Leukocyte trans-endothelial migration (hsa04670), Oocyte meiosis (hsa04114), Progesterone-mediated oocyte maturation (hsa04914), Chemokine signaling pathway (hsa04062), Intestinal immune network for IgA production (hsa04672), Axon guidance (hsa04360) and Cytokine-cytokine receptor interaction (hsa04060). On the other hand, reactome highlighted eighteen pathways, with the same statistical conditions intact. The major pathways highlighted were formation of the cornified envelope (HSA-6809371), developmental biology (HSA-1266738), immune system (HSA-168256) and cell cycle, mitotic (HSA-69278).
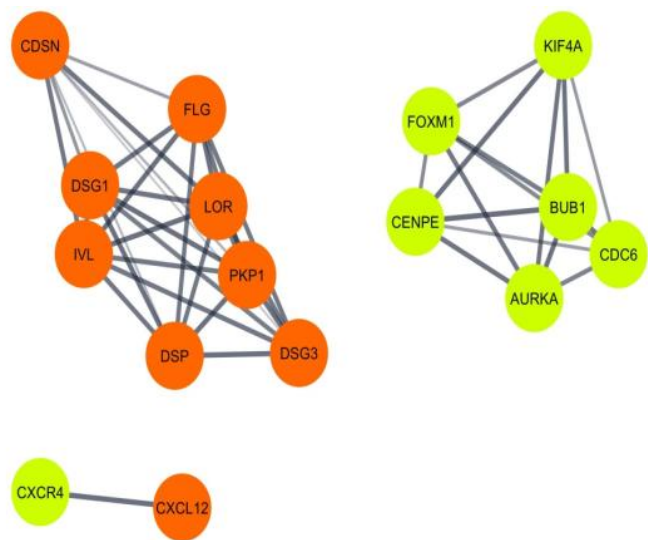

Fig. 2. PPI Network of the hub genes with 16 nodes and 34 edges. The 16 hub genes were used to construct a network using STRING database in Cytoscape. The 'orange' nodes specify the downregulated genes while the 'green' nodes specify upregulated genes.

\section{E. Target gene prediction of DE microRNA}

A total of 55,684 target interactions (experimental and predictive) were identified as putative targets for the 17 DEmiRs gathered in this study. The data acquired was then used to identify validated and predictive pairs of DEGsDEmiRs from this study. Out of a possible of 956 individual genes, 28 genes were found to be overlapping with the DEGs as well. These include ABHD5, CA12, CD164, CDH1, CLIP1, COLEC12, CXCR4, DEPDC1, EGLN3, ELAVL2, EREG, HIPK3, IGF1, IGF2BP3, KLF4, KLF5, MBNL1, MBNL2, NAB1, PICALM, RECK, RORA, SOX9, TM4SF1, TWF1, VAMP3, VSNL1 and WDR1. 56 pairs of validated DEGs-DEmiRs and 221 pairs of predicted DEGsDEmiRs were curated from miRTarBase and TargetScan \& miRDB respectively.

\section{F. Construction and analysis of DEG-DEmiR network}

The DEGs and DEmiRs were used towards constructing a network using Cytoscape which consisted of 47 nodes and 267 edges. Using Network Analyser tool of Cytoscape, the above network was analysed for the purpose of finding hub miRNAs. A degree cut-off of $>20$ was set as the criteria, and subsequently, three major hubs identified were hsa-miR-19a, hsa-miR-9-5p and hsa-miR-1. The hubs were primarily enriched in positive regulation of transcription, positive 
regulation of mitotic nuclear division, cadherin binding involved in cell-cell adhesion, positive regulation of fibroblast proliferation, protein kinase B signalling and pathways in cancer.
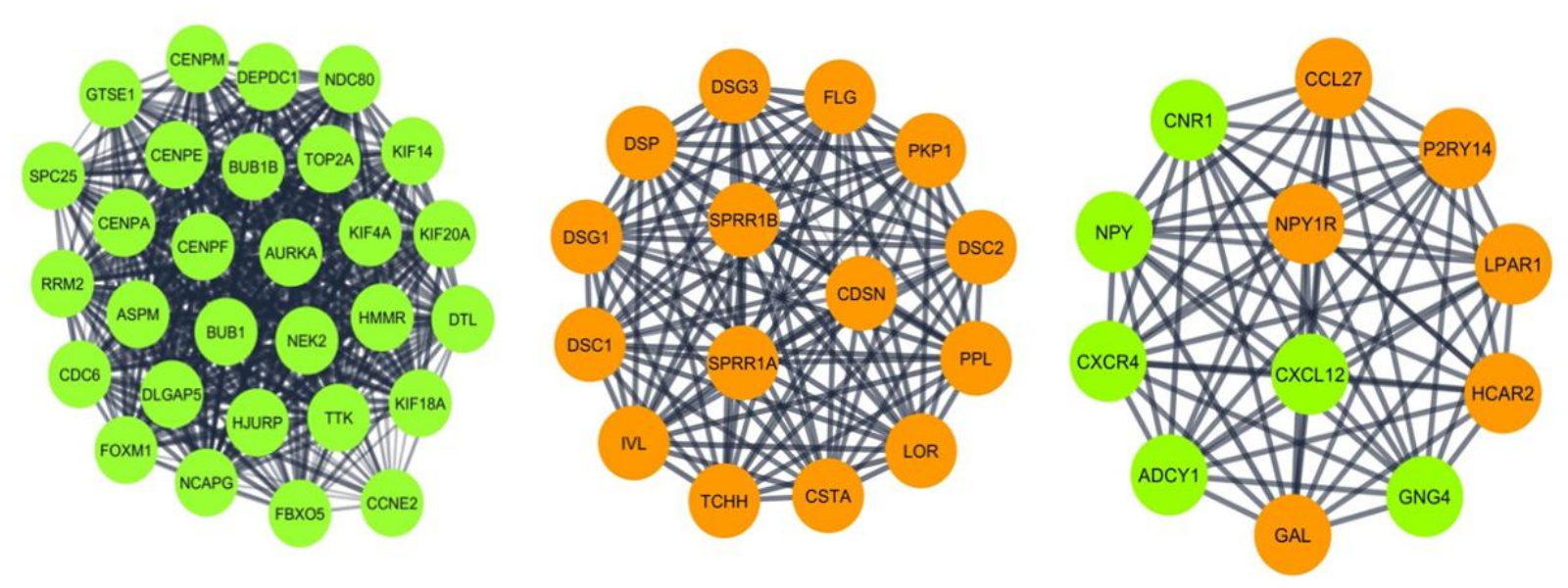

Fig. 3. Significant clusters identified from PPI using MCODE plugin. The circular orange nodes represent downregulated genes while the circular green nodes represent upregulated genes.

\section{VALIDATION}

According to Human Protein Atlas (HPA), genes FLG, DSG1, LOR, CDSN and IVL show aberrant expression in skin cancers in comparison to normal skin samples. Other Critical genes were validated through disease association microarray study approach. There are only two drugs approved by FDA namely Avelumab (trade name Bavencio) and Pembrolizumab (trade name Keytruda) for Merkel cell carcinoma. Both of these drugs are antibody that targets the programmed death-ligand 1 (PD-L1) and programmed cell death protein 1 (PD-1) respectively. PD-1 is the superfamily of immunoglobulin that binds to PD-L1 and PD-L2. Using STRING database, gene CXCR4 is showing interaction with PDCD1 and CXCL12 has interaction with CXCR4 gene. To validate rest of the genes we used micoarray analysis approch. 3 microarray datasets are used for our validation namely GSE22396, GSE50451 and GSE104869. These datasets are first normalised using $\mathrm{R}$ and then further analysed using Altanalyze (version 2.1.3). GSE22396 and GSE50451 microarray dataset are related to Merkel Cell Carcinoma (MCC) analysis whereas GSE104869 is the microarray of Vemurafenib drug which is used in late stage of melanoma. In GSE22396 and GSE50451 microarray fold change of upregulated genes are positive and downregulated genes are negative shown in Table 3. In GSE104869 gene expression before and after the drug usage is employed to find out the 'fold change' of our critical genes and fold change showing the negative values in upregulated genes indicating that these genes might be responsible for Merkel Cell Carcinoma (MCC).
TABLE III. A TABULAR REPRESENTATION SHOWING FOLD CHANGE VALUES IN DIFFERENT MICROARRAY DATASET GSE22396, GSE50451 AND GSE104869. GREEN AND RED COLOUR REPRESENTS UPREGULATED AND DOWNREGULATED GENES RESPECTIVELY.

\begin{tabular}{|l|l|l|l|}
\hline $\begin{array}{c}\text { Gene } \\
\text { symbol }\end{array}$ & $\begin{array}{c}\text { GSE22396 (Log } \\
\text { FC) }\end{array}$ & $\begin{array}{c}\text { GSE50451 (Log } \\
\text { FC) }\end{array}$ & \multicolumn{1}{|c|}{$\begin{array}{c}\text { GSE104869 (Log } \\
\text { FC) }\end{array}$} \\
\hline AURKA & 0.48525592 & 0.19211608 & -0.69955388 \\
\hline KIF4A & 0.49459202 & 0.07702051 & -0.76814444 \\
\hline PKP1 & -1.18842001 & 0.09340696 & Not found \\
\hline BUB1 & 0.65614758 & 0.09906461 & -0.85706888 \\
\hline CDC6 & 0.3905455 & 0.06685074 & -0.80892104 \\
\hline CENPE & 0.62753201 & 0.0191033 & -0.9252193 \\
\hline FOXM1 & 0.65953432 & 0.03433299 & -0.70192919 \\
\hline DSG3 & -1.86577326 & 0.11021616 & Not found \\
\hline DSP & -1.60072796 & 0.08411476 & -0.68181818 \\
\hline
\end{tabular}

\section{CONCLUSION}

MCC is an aggressive form of neuroendocrine carcinoma that represents the second most common cause of skin cancer related deaths. MCC is often linked with a weak prognosis, as more than $33.33 \%$ of patients die from the disease compared to $\sim 15 \%$ for malignant melanoma. Due to the rarity of the disease and of the occurrence of Merkle cells, MCC remains a cancer type that is yet to be fully researched and understood. This is of immediate consequence now as the incidences of MCC have been on a rise and the disease is often associated with fatality. In fact, only two FDA approved drugs exist in the market for treatment of MCC. Further, there is a lack of definitive biomarkers that could aid in a more effective selection of patients for new therapies, thereby creating a crucial need for further research The current study identified hub genes and hub miRNAs associated with MCC using integrated bioinformatics along 
with their functional analysis, PPI network construction, module selection and module enrichment analyses. Out of these 16 genes either downregulated or upregulated, 6 gene are further validated by microarray analysis. For validation three microarrays are used, two microarrays are related to Merkel Cell Carcinoma (MCC) and one microarray is for Vemurafenib drug which is FDA approved drug for Merkel Cell Carcinoma. After that several related literatures is also studied for validation of critical gene for carcinoma. In conclusion, the findings of the current work shows that gene found by these tools are responsible for MCC and provide prerequisite for future studies to identify useful markers and understand underlying mechanisms related to MCC carcinogenesis.

\section{ACKNOWLEDGMENT}

Authors express their acknowledgements to Rajkumar Chakraborty and Mehak Bhatnagar.

\section{REFERENCES}

[1] Schadendorf D, Lebbe C, Zur Hausen A, et al. (2017). Merkel cell carcinoma: epidemiology, prognosis, therapy and unmet medical needs. Eur J Cancer, 71, 53-69.

[2] Tang CK, Toker C. (1978). Trabecular carcinoma of the skin: an ultrastructural study. Cancer, 42(5), 2311-21.

[3] Kaae J, Hansen AV, Biggar RJ, Boyd HA, Moore PS, Wohlfahrt J, et al. (2010). Merkel cell carcinoma: incidence, mortality, and risk of other cancers. J Natl Cancer Inst, 102 (11), 793-801.
[4] Kaufman HL, Russell J, Hamid O, Bhatia S, Terheyden P, D'Angelo SP, et al. (2016). Avelumab in patients with chemotherapy refractory metastatic Merkel cell carcinoma: A multicentre, single-group, openlabel, phase 2 trial. Lancet Oncol, 17(10), 1374-85.

[5] Nghiem PT, Bhatia S, Lipson EJ, Kudchadkar RR, Miller NJ, Annamalai L, et al. (2016). PD-1 blockade with Pembrolizumab in advanced Merkel-cell carcinoma. N Engl J Med, 374(26), 2542-52.

[6] Huang X, Yuan T, Liang M, Du M, Xia S, Dittmar R, Wang D, See W, Costello BA, Quevedo F, Tan W, Nandy D, Bevan GH, Longenbach S, Sun Z, Lu Y, Wang T, Thibodeau SN, Boardman L, Kohli M, Wang L. (2014). Exosomal miR-1290 and miR-375 as prognostic markers in castration-resistant prostate cancer. Eur Urol, 67(1), 3-41.

[7] Szklarczyk D, Gable AL, Lyon D, Junge A, Wyder S, Huerta-Cepas J, Simonovic M, Doncheva NT, Morris JH, Bork P, Jensen LJ, Mering CV. (2019). STRING v11: protein-protein association networks with increased coverage, supporting functional discovery in genome-wide experimental datasets. Nucleic Acids Res., 47(D1):D607-D613.

[8] Bader GD, Hogue CW. (2003). An automated method for finding molecular complexes in large protein interaction networks. BMC Bioinformatics, 13; 4:2.

[9] Aytes, Alvaro et al. "Cross-species regulatory network analysis identifies a synergistic interaction between FOXM1 and CENPF that drives prostate cancer malignancy." Cancer cell vol. 25,5 (2014): 638651. doi:10.1016/j.ccr.2014.03.017.

[10] Becker JC. Merkel cell carcinoma. (2010). Ann Oncol, 21(7), 81-85.

[11] Burack J, Altschuler EL. (2003). Sustained remission of metastatic Merkel cell carcinoma with treatment of HIV infection. J R Soc Med. 9(6), 238-239. doi: 10.1258/jrsm.96.5.238.

[12] Mariot P, Vanoverberghe K, Lalevee N, Rossier MF \& Prevarskaya N. (2002). Overexpression of an alpha $1 \mathrm{H}$ (CaV3.2) T-type calcium channel during neuroendocrine differentiation of human prostate cancer cells. J. Biol. Chem, 277(13), 10824-10833. 